$\mathrm{T} 03 / 133$

hep-th/0309117

\title{
Detuned Branes and Supersymmetry Breaking
}

\author{
Ph. $\operatorname{Brax}^{1 a}$, N. Chatillon ${ }^{2 a}$ \\ ${ }^{a}$ Service de Physique Théorique \\ $C E A / D S M / S P h T$, Unité de recherche associée au CNRS, \\ CEA-Saclay F-91191 Gif/Yvette cedex, France
}

\begin{abstract}
We consider the spontaneous breaking of supersymmetry in five dimensional supergravity with boundaries where the supersymmetry breaking mechanism is provided by the even part of the bulk prepotential. The supersymmetric action comprises boundary brane terms with detuned tensions. The two branes have opposite tensions. We analyse the possible vacua with spontaneously broken supersymmetry. A class of solutions corresponds to rotated branes in an $A d S_{5}$ bulk. In particular parallel branes which are rotated with respect to the bulk preserve $1 / 4$ of supersymmetry. We analyse more general vacua using the low energy effective action for gravity coupled to the radion field. Supersymmetry breaking implies that the radion field acquires a potential which is negative and unbounded from below. This potential is modified when coupling the boundary branes to a bulk four-form field. For a brane charge larger than the deficit of brane tension, the radion potential is bounded from below while remaining flat when the charge equals the deficit of brane tension.
\end{abstract}

\section{Introduction}

Supersymmetry breaking is one of the stumbling blocks of physics beyond the standard model. String inspired models suffer from the impossibility of breaking supersymmetry at the perturbative level [1. Non-perturbative mechanisms such as gaugino condensation provide a useful framework [2], although one has to resort to race-track potentials which are not free from fine-tuning problems [3]. An appropriate way of breaking supersymmetry would have to face stringent

\footnotetext{
${ }^{1}$ brax@spht.saclay.cea.fr

${ }^{2}$ chatillon@spht.saclay.cea.fr
} 
experimental constraints springing from both particle physics and cosmology. In cosmology, the cosmological constant issue requires to adjust the vacuum energy to almost zero energy compared to the Planck scale [4. This is difficult to reconcile with supergravity breaking where one expects a vacuum energy of the order $O\left(m_{3 / 2}^{2} M_{\mathrm{Pl}}^{2}\right)$. In particle physics, one expects that the non-observation of superpartners is due to a large mass splitting of the order of one $\mathrm{TeV}$ within supersymmetry multiplets.

Recently a new paradigm for supersymmetry breaking has been provided by extra-dimensional models [5]. In particular D-branes [6] allow new mechanisms of supersymmetry breaking. For instance supersymmetry breaking may be transmitted from one brane to another by bulk fields leading to hierarchical energy scales [7. One can also design brane anti-brane configurations which break all supersymmetry [8, 9, 10, and non-supersymmetric branes which tend to be unstable objects [8, 11]. A $N=1$ superspace formulation of $5 \mathrm{~d}$ supergravity in a flat background with boundary branes has been recently presented [12.

In this paper we will focus on a simpler framework, i.e. the supersymmetric Randall-Sundrum model where bulk supergravity is coupled to two boundary branes [13, 14, 15, 16, 17, 18, 19, 20, 21, 10, 22, 23, 24, 25, 26]. In particular we will be interested in spontaneously broken supersymmetry whereby branes become tilted, i.e branes which are not hyperplanes at constant $x_{5}$ when the bulk metric reads

$$
d s^{2}=d x_{5}^{2}+a^{2}\left(x_{5}\right) \eta_{\mu \nu} d x^{\mu} d x^{\nu} .
$$

In section 2 we will consider the supersymmetric Randall-Sundrum model spontaneously broken by the even part of the bulk prepotential [10. This amounts to detuning the tensions of the boundary branes by a factor $T<1$. In section 3 we present a class of solutions representing rotated branes. These branes are hyperplanes at angles. In the non-singular case of rotated and parallel brane configurations, Lorentz invariance is broken down to $S O(1,2)$. In section 4 we find the Killing spinors for parallel branes preserving $1 / 4$ of supersymmetry. We also consider the massive gravitini showing that they do not survive due to the incompatibility between the boundary conditions on the branes and the propagation in the bulk. Finally in section 5 we analyse supersymmetry breaking from the four dimensional point of view using the low energy effective action. This allows one to discuss generic supersymmetry breaking vacua. We find that the radion potential is negative and unbounded from below. Hence cosmological solutions all end up in a big crunch singularity. We also discuss non-singular static configurations. Finally we couple the boundary branes to a bulk fourform field. One finds a BPS bound linking the charge to the deficit of brane tension. When the charge exceeds the BPS bound the radion potential becomes bounded from below with a vacuum corresponding to infinitely far apart branes. The BPS case when the charge equals the deficit of brane tension leads to a vanishing potential for the radion field. In section 6 we give some comments and conclusions. We have also included an appendix where the equivalence between a single $A d S_{4}-$ brane and a rotated brane has been made explicit. Another 
appendix deals with details about massless gravitons and rotated branes.

\section{Supergravity with Tilted Boundary Branes}

\subsection{Boundary conditions on tilted branes}

We extend the known supersymmetrization of the Randall-Sundrum model [16] to the case where the boundary branes are tilted. In particular we deduce a supersymmetric Lagrangian and associated boundary conditions which are compatible with arbitrarily curved boundary branes. The issue of supersymmetry breaking by the tilting of the boundary branes will be tackled in section 3 .

Let us consider pure supergravity in the bulk comprising a supergravity multiplet containing the metric $g_{a b}, a, b=1 \ldots 5$, the gravitini $\psi_{a}^{A}$ where $A=$ 1,2 is a symplectic index (the gravitini are Majorana-symplectic spinors), and finally the graviphoton $A_{a}$. The graviphoton can be used as the gauge field when gauging a $U(1)_{R}$ subgroup of the $S U(2)_{R}$ symmetry. Such a gauging implies the presence of a cosmological constant $-6 k^{2}$ in the bulk and modifies the supersymmetry variations (with the normalization of [27])

$$
\delta \psi_{a}^{A} \supset D_{a} \epsilon^{A}-\frac{i \sqrt{2}}{3} \mathcal{P}^{A B} \gamma_{a} \epsilon_{B}
$$

The bulk bosonic action reduces to the $A d S_{5}$ action

$$
S=\frac{1}{\kappa_{5}^{2}} \int d^{5} x \sqrt{-g}\left(\frac{1}{2} R+6 k^{2}\right)
$$

admitting a bulk solution of the equations of motion in the form [13]

$$
d s^{2}=d x_{5}^{2}+a^{2}\left(x_{5}\right) \eta_{\mu \nu} d x^{\mu} d x^{\nu}
$$

where $a\left(x_{5}\right)=e^{-k x_{5}}$ is the warp factor.

We would like to define supergravity on a $Z_{2}$ orbifold with arbitrary boundary branes. To simplify matters we assume that the boundary branes are located at

$$
x_{5}^{1}=\xi_{1}\left(x^{\mu}\right), x_{5}^{2}=\rho+\xi_{2}\left(x^{\mu}\right)
$$

where $\xi_{1,2}$ are infinitesimal displacements.

In order to define a covering space it is convenient to perform a change of coordinates which straightens up both branes defining

$$
\hat{x}^{5}=x^{5}+\xi^{5}\left(x^{5}, x^{\mu}\right)
$$

where the function $\xi^{5}$ must satisfy

$$
\xi^{5}\left(\xi_{1}, x^{\mu}\right)=-\xi_{1}, \xi^{5}\left(\rho+\xi_{2}, x^{\mu}\right)=-\xi_{2} .
$$

The resulting branes are located at

$$
\hat{x}_{1}^{5}=0, \hat{x}_{2}^{5}=\rho .
$$


The simplest possibility for $\xi^{5}$ is provided by a linear function in $x^{5}$. One must accompany the change in $x^{5}$ with a small change of four dimensional coordinates 28

$$
\hat{x}^{\mu}=x^{\mu}-\eta^{\mu \nu}\left(\int_{0}^{x^{5}} \frac{d u}{a^{2}(u)} \partial_{\nu} \xi^{5}\left(u, x^{\lambda}\right)\right) \equiv x^{\mu}+\xi^{\mu} .
$$

Such a transformation implies that the metric is not in a normal Gaussian form anymore

$$
\hat{g}_{55}=1-2 \partial_{5} \xi_{5}
$$

where $\xi_{5}=\xi^{5}$ and

$$
\hat{g}_{\mu \nu}=a^{2}\left[\left(1+2 k \xi^{5}\right) \eta_{\mu \nu}+2\left(\int \frac{d u}{a^{2}(u)} \partial_{\mu} \partial_{\nu} \xi^{5}\left(u, x^{\lambda}\right)\right)\right] .
$$

The main property of the change of coordinates where the branes are straightened up is that

$$
\hat{g}_{\mu 5}=0 .
$$

Defining the radion as the distance between the boundary branes

$$
R=\int_{0}^{\rho} \sqrt{\hat{g}_{55}} d x_{5}=\rho+\xi_{2}-\xi_{1}
$$

one finds that it is independent of the choice of $\xi^{5}$.

We can now define the $Z_{2}$ action in the $\left(\hat{x}^{5}, \hat{x}^{\mu}\right)$ coordinates by compactifying the $\hat{x}^{5}$ coordinate on a circle of perimeter $2 \rho$ and identifying $\left(\hat{x}^{5}, \hat{x}^{\mu}\right) \equiv$ $\left(-\hat{x}^{5}, \hat{x}^{\mu}\right)$. The two boundary branes are now fixed points under the $Z_{2}$ action.

In the non-gaussian coordinate system, one can impose the usual boundary conditions on the fields which acquire the usual parity under the $Z_{2}$ action. Thus $\hat{g}_{\mu \nu}$ and $\hat{g}_{55}$ are even, $\hat{g}_{\mu 5}$ is odd, $\hat{A}_{\mu}$ is odd while $\hat{A}_{5}$ is even. The gravitini have particular boundary conditions

$$
\left(\delta_{B}^{A}-\Gamma \mathcal{Q}_{B}^{A}\right) \hat{\psi}_{\mu}^{B}=0
$$

and

$$
\left(\delta_{B}^{A}+\Gamma \mathcal{Q}_{B}^{A}\right) \hat{\psi}_{5}^{B}=0
$$

on both branes where we have introduced

$$
\Gamma=-n^{a} \gamma_{a}
$$

and $n^{a}$ is the normalized normal vector to the brane whose fifth component is chosen to be negative. We have also used a matrix $\mathcal{Q}_{B}^{A}$ in the Lie algebra of $S U(2)$. Finally, the odd fields $\hat{g}_{\mu 5}$ and $\hat{A}_{\mu}$ vanish on the branes.

We can now perform the inverse change of coordinates going back to the situation where the branes are tilted and the bulk admits Gaussian normal coordinates. As already stated the $g_{\mu 5}$ component of the metric vanishes in both frames. This leads to no restriction on the metric from the $Z_{2}$ symmetry. We will focus on the boundary conditions for the gravitini in the supergauge 
$A_{5}=0$ and $\psi_{5}^{A}=0$. Indeed we will analyse the mass spectrum of KaluzaKlein states for the gravitini using this constraint in section 4 . The boundary condition of the graviphoton on the brane becomes

$$
\left.A_{\mu}\right|_{\text {brane }}=0
$$

while the two boundary conditions for the gravitini become

$$
\left.\left(\delta_{B}^{A}-\Gamma \mathcal{Q}_{B}^{A}\right) \psi_{\mu}^{B}\right|_{\text {brane }}=0
$$

supplemented with

$$
\left.\left(\partial_{5} \xi^{\nu}\right) \psi_{\nu}^{A}\right|_{\text {brane }}=0 .
$$

Here the normal vector reads

$$
n_{1,2}^{a}=\frac{1}{\sqrt{\tilde{n}_{a}^{1,2} g^{a b} \tilde{n}_{b}^{1,2}}} \tilde{n}_{1,2}^{a}
$$

where

$$
\tilde{n}_{a}^{1,2}=\left(\partial_{\mu} \xi_{1,2},-1\right) .
$$

These boundary conditions will be crucial in determining the spectrum of gravitini. Finally the boundary condition for the spinors parametrizing the supersymmetric variations is

$$
\left(\delta_{B}^{A}-\Gamma \mathcal{Q}_{B}^{A}\right) \epsilon^{B}=0
$$

on the brane.

\subsection{Supersymmetric action}

Let us come back to the supersymmetrization of the brane and bulk system. To do that we need to specify the prepotential in terms of even and odd functions under the $Z_{2}$ symmetry [10]

$$
\mathcal{P}^{A B}=i\left(\frac{9}{8}\right)^{1 / 2}\left[\epsilon\left(\hat{x}_{5}\right) k T \mathcal{Q}^{A B}+k \sqrt{1-T^{2}} \Sigma^{A B}\right]
$$

where the matrices $\mathcal{Q}$ and $\Sigma$ anticommute $\{\mathcal{Q}, \Sigma\}=0$ and $\mathcal{Q}^{2}=1, \Sigma^{2}=1$, together with the Lie algebra properties of hermiticity and tracelessness. The function $\epsilon\left(\hat{x}_{5}\right)$ jumps from -1 to 1 at the origin and from 1 to -1 at $\rho$.

The variation of the action under a supersymmetry transformation of spinor $\epsilon^{A}$ leads to boundary terms from the gravitino kinetic terms. More precisely the action of the fifth derivative on the odd part of the prepotential leads to delta-functions which can be compensated by the variation of the boundary action [10, 22]

$$
S=-\frac{6 k T}{\kappa_{5}^{2}} \int_{1} d^{4} x \sqrt{-h}+\frac{6 k T}{\kappa_{5}^{2}} \int_{2} d^{4} x \sqrt{-h}
$$

where $h_{\mu \nu}$ is the induced metric on the brane. The brane tensions are constant and opposite

$$
\lambda_{ \pm}= \pm \frac{6 k T}{\kappa_{5}^{2}}
$$


Notice that the factor $T<1$ introduced in the prepotential and parameterizing its even part leads to a detuning of the brane tension with respect to the tuned Randall-Sundrum case $T=1$. More general detunings in supergravity have been considered in [22].

The action comprising the bulk supergravity Lagrangian and the boundary brane terms is supersymmetric. We will see that as soon as $T<1$ supersymmetry is spontaneously broken. The boundary brane action with $T<1$ implies that static branes sitting at constant $x_{5}$ in the bulk are no longer vacua of the theory.

\section{Rotated Branes and Lorentz Invariance Vio- lation}

\subsection{Rotated branes}

One can easily find a class of vacua of the spontaneously broken models $T<1$. Going to conformal coordinates

$$
d s^{2}=a^{2}(y)\left(d y^{2}+\eta_{\mu \nu} d x^{\mu} d x^{\nu}\right)
$$

in the bulk where $a(y)=1 / k y$, one can accomodate the change of boundary conditions induced by the detuning of the brane tensions by rotating the boundary branes with respect to the $y$ axis. Decompose four dimensional vectors according to an orthogonal basis $\left(h^{\mu}, e_{i}^{\mu}\right)$ where $h^{\mu}$ is space-like and $e_{i}^{\mu} e_{j}^{\nu} \eta_{\mu \nu}=\eta_{i j}$. For instance $^{3}$ we identify $x^{\mu}=X \frac{h^{\mu}}{\sqrt{h^{2}}}+X^{i} e_{i}^{\mu}$ and $d x^{\mu} d x_{\mu}=-d X_{0}^{2}+d X_{1}^{2}+d X_{2}^{2}+d X^{2}$. Consider the rotation

$$
\begin{aligned}
\hat{y} & =\frac{y-\sqrt{h^{2}} X}{\sqrt{1+h^{2}}} \\
\hat{X} & =\frac{X+\sqrt{h^{2}} y}{\sqrt{1+h^{2}}}
\end{aligned}
$$

where the angle of rotation $\theta$ satisfies $\tan \theta=\sqrt{h^{2}}$. We leave $\left(X_{0}, X_{1}, X_{2}\right)$ invariant. This leads to the metric

$$
d s^{2}=a^{2}\left(\frac{\hat{y}+\sqrt{h^{2}} \hat{X}}{\sqrt{1+h^{2}}}\right)\left(d \hat{y}^{2}-d X_{0}^{2}+d X_{1}^{2}+d X_{2}^{2}+d \hat{X}^{2}\right)
$$

in the bulk. One of the boundary branes located at $(\hat{y}=$ constant $)$ satisfies the Israel boundary conditions

$$
\left.\frac{\partial_{\hat{y}} a}{a^{2}}\right|_{\hat{y}=\text { constant }}=-\frac{k}{\sqrt{1+h^{2}}}=-k T
$$

\footnotetext{
${ }^{3}$ We denote by dot the Minkowski scalar product; similarly $h^{2}$ is defined as $h_{\mu} \eta^{\mu \nu} h_{\nu}$.
} 
provided the tilting vector $h^{\mu}$ fulfills

$$
h^{2}=\frac{1}{T^{2}}-1 .
$$

Notice that this vector is indeed space-like. Perform then the inverse rotation. The brane is located at $y=h . x+$ constant in conformal coordinates $\left(x^{\mu}, y\right)$. Apply the same procedure to the second boundary brane. We thus find that the two boundary branes depend on two tilting vectors $h_{1,2}^{\mu}$ with the same norm. We choose the positions of the branes to be at

$$
y=h_{1} . x+\frac{1}{k}, y=h_{2} . x+\frac{e^{k \rho}}{k} .
$$

The constants have been chosen for convenience. The branes are hyperplanes in conformal coordinates. They correspond to rotated branes which are moving if $h_{0} \neq 0$. In the following we will refer to this brane configuration as rotated branes. We will be particularly interested in the parallel configurations

$$
y=h . x+\frac{1}{k}, y=h . x+\frac{e^{k \rho}}{k} .
$$

where $h=h_{1}=h_{2}$. Such configurations are not perturbatively connected to the usual boundary branes of the Randall-Sundrum model located at $y=$ const. Indeed the distance between the branes, i.e. the radion, is

$$
R(x)=\frac{1}{k} \ln \left(\frac{h \cdot x+\frac{e^{k \rho}}{k}}{h \cdot x+\frac{1}{k}}\right)
$$

which goes to infinity on the planes where the two branes reach $y=0$.

As $T<1$ one might have expected to find the $A d S_{4}$ brane configurations of [29]. This is only possible if the two boundary branes carry two tensions of the same sign. Here the opposite sign of the tensions forces us to consider two rotated branes with the same norm $h_{1}^{2}=h_{2}^{2}$ [10] (see appendix A for more details).

Let us examine the geometry of one of the boundary branes in more detail. Notice first that the boundary branes hit the boundary $y=0$ along an codimension two plane. Focus on the positive tension brane. This happens for $h_{1} \cdot x+\frac{1}{k}=0$. For convenience sake, let us focus on the case where $h_{1}$ lies along the third axis only. The induced metric reads

$$
d s_{4}^{2}=d z^{2}+e^{-2 \sqrt{1-T^{2}} k z}\left(-d t^{2}+d x_{1}^{2}+d x_{2}^{2}\right)
$$

where we have identified

$$
k \sqrt{1-T^{2}} z=\ln \left(k h_{1} x_{3}+1\right)
$$

Notice that such a coordinate system is only valid on one of the branes at a time and not globally. When the brane hits the boundary at $y=0$, the coordinate $z \rightarrow-\infty$. Moreover $z$ covers the whole real line. Using the coordinates 
$\left(t, x_{1}, x_{2}, z\right)$ the boundary geometry does not present any pathological behaviour and is simply identified with $A d S_{4}$ with cosmological constant

$$
\Lambda_{A d S_{4}}=-3 k^{2}\left(1-T^{2}\right)
$$

The same analysis applies to the second brane of negative tension.

There are three typical global configurations that one may envisage for the two brane system. First of all the branes can intersect and the supergravity description breaks down at the intersection hyperplane. This is the generic case. One may also consider the case where the would-be intersection is beyond the $A d S_{5}$ boundary located at $y=0$. Finally one may send the would-be intersection to infinity by choosing the two branes to be parallel. In the following we will focus on the parallel case.

\subsection{Lorentz invariance violation}

One can use either the description in terms of rotated branes or change coordinates to non-rotated boundary branes with a space-time dependent metric. In order to study the violation of Lorentz invariance it is convenient to use the non-rotated boundary brane picture after the change of coordinates

$$
\hat{x}^{5}=\frac{\rho}{\rho+\xi_{2}-\xi_{1}}\left(x^{5}-\xi_{1}\right) .
$$

For small brane tilting $\xi_{1,2}$ this corresponds to

$$
\xi^{5}=\frac{\left(\xi_{1}-\xi_{2}\right)}{\rho} x_{5}-\xi_{1}
$$

where (31) leads to

$$
\xi_{1}=h . x, \xi_{2}=e^{-k \rho} h . x .
$$

for small $\xi_{1,2}$ and small enough $x$. The metric is not in the normal Gaussian form anymore

$$
\hat{g}_{55}=1+2 \frac{\xi_{2}-\xi_{1}}{\rho}
$$

and

$$
\hat{g}_{\mu \nu}=a^{2}\left(1+2 k \xi_{5}\right) \eta_{\mu \nu} .
$$

This implies that the background can be described as obtained from the RandallSundrum background by switching on a non-trivial vev for the radion field

$$
<R>(x)=\rho+\xi_{2}(x)-\xi_{1}(x) .
$$

This is an explicitly Poincaré violating effect as it is $x^{\mu}$-dependent. More precisely, let us perform a Lorentz transformation

$$
\tilde{x}^{\mu}=x^{\mu}+M^{\mu \nu} x_{\nu}
$$


where $M^{\mu \nu}$ is antisymmetric. The bulk metric is invariant provided

$$
M_{\nu}^{\mu} h^{\nu}=0 .
$$

The Lorentz group is broken to $S O(1,2)$. Similarly translation invariance $\tilde{x}^{\mu}=$ $x^{\mu}+a^{\mu}$ implies that $a_{\mu} h^{\mu}=0$, i.e. momenta $p^{\mu}$ are only defined in the plane orthogonal to $h$

$$
p_{\mu} h^{\mu}=0 .
$$

The Poincaré invariance of the bulk metric is the three dimensional Poincaré group.

Another way of realizing that Poincaré invariance is broken springs from the induced metric (34) on one of the boundary branes. The boundary metric of $A d S_{4}$ admits a $S O(2,3)$ invariance with a $S O(1,2)$ subgroup of linearly acting Lorentz transformations.

\subsection{Brane supersymmetry breaking}

As well as Lorentz invariance violation in the bulk, supersymmetry on the branes is also affected. We consider brane matter in the form of four dimensional chiral superfields $\Phi$ living on the positive tension brane. The coupling of brane matter to the radion superfield springs from the Kähler potential [20]

$$
K=-3 \ln \left(1-e^{-k(S+\bar{S})}-|\Phi|^{2}\right)
$$

where $\Phi$ is dimensionless and $S+\bar{S}=2 R$. Now the kinetic terms of the radion field in the Einstein frame reads

$$
L_{k i n}=K_{S \bar{S}}\left|\frac{\partial S}{\partial x}\right|^{2} .
$$

This leads to a soft supersymmetric breaking mass for the chiral multiplets

$$
m_{\Phi}^{2}=6 k^{2} e^{-k(S+\bar{S})}\left|\frac{\partial S}{\partial x}\right|^{2}
$$

for large $R$. More explicitly, the soft breaking mass reads

$$
m_{\Phi}^{2}=6 k^{2} \frac{1-T^{2}}{T^{2}} e^{-2 k<R>} .
$$

As expected this mass term is Poincaré violating due to the explicit dependence of $\langle R>$ on the space-time coordinates.

\subsection{Massless gravitons}

We will analyse the influence of the breaking of Poincaré invariance on massless gravitons. Let us first state the Israel junction condition on both branes

$$
K_{\mu \nu}-h_{\mu \nu} K= \pm 3 T k h_{\mu \nu}
$$


where $h_{\mu \nu}$ is the induced metric on the branes. This implies that

$$
K_{\mu \nu}=\mp T k h_{\mu \nu} .
$$

It is convenient to introduce the vielbein $e_{\mu}^{a}=\frac{\partial Y^{a}}{\partial x^{\mu}}$ where $Y^{a}$ is the embedding of the branes in conformal coordinates. In our case the only non-zero components are

$$
e_{\mu}^{a}=\delta_{\mu}^{a}, e_{\mu}^{5}=\partial_{\mu} Y^{5} \equiv h_{\mu} .
$$

The extrinsic curvature tensor is defined by

$$
K_{\mu \nu}=e_{\mu}^{a} e_{\nu}^{b} D_{a} n_{b} .
$$

The perturbed metric is chosen in the bulk

$$
d s_{5}^{2}=a^{2}(y)\left(d y^{2}+\left(\eta_{\mu \nu}+n_{\mu \nu}\left(x^{\lambda}, y\right)\right) d x^{\mu} d x^{\nu}\right) .
$$

This implies that to first order in $n_{\mu \nu}$

$$
n=a A\left(h_{\mu},-1\right)
$$

where

$$
A=\frac{1}{\sqrt{1+h_{\mu} \eta^{\mu \nu} h_{\nu}}}\left(1+\frac{1}{2} \frac{h_{\mu} n^{\mu \nu} h_{\nu}}{1+h_{\nu} \eta^{\mu \nu} h_{\nu}}\right) .
$$

The perturbed Israel junction conditions to first order in $n_{\mu \nu}$ read

$\partial_{5} n_{\mu \nu}+h_{\mu} \partial_{5} n_{\nu}^{\rho} h_{\rho}+h_{\nu} \partial_{5} n_{\mu}^{\rho} h_{\rho}+h_{\lambda}\left(\partial_{\mu} n_{\nu}^{\lambda}+\partial_{\nu} n_{\mu}^{\lambda}-\partial^{\lambda} n_{\mu \nu}\right)=a k \frac{h_{\mu} n^{\mu \nu} h_{\nu}}{1+h_{\mu} \eta^{\mu \nu} h_{\nu}}\left(\eta_{\mu \nu}+h_{\mu} h_{\nu}\right)$

where we have explicitly used $\partial_{\mu} h^{\nu}=0$.

A sufficient way of satisfying the junction conditions is to impose Neumann boundary conditions

$$
n^{a} \partial_{a} n_{\mu \nu}=0
$$

on the branes. In the bulk the graviton modes read

$$
n_{\mu \nu}\left(y, x^{\mu}\right)=\phi(y) e^{i p . x} n_{\mu \nu}
$$

where $p^{2}=0$ for massless excitations and $n_{\mu \nu}$ is transverse and traceless. In the massless case $\phi(y)$ is constant so that the Neumann boundary condition is automatically satisfied provided that

$$
p \cdot h=0
$$

corresponding to the absence of translation invariance in the normal direction to the brane. The boundary condition implies then that

$$
h^{\mu} n_{\mu \nu}=0 .
$$


This is tantamount to saying that gravitons must be polarised along the brane, i.e. orthogonal to the brane normal vector. The necessary and sufficient analysis of the boundary conditions is detailed in Appendix B.

The condition $\partial_{\mu} h_{\nu}=0$ breaks the residual gauge symmetry $x^{\mu} \rightarrow x^{\mu}+\epsilon^{\mu}$ where $\square \epsilon^{\mu}=0$ to preserve the transversality of the metric perturbation and $\partial_{c} \epsilon^{c}=0$ to preserve its tracelessness. We have to impose

$$
\epsilon . h=0
$$

reducing the number of gauge degrees of freedom.

The tensor $n_{\mu \nu}$ has five independent components. Let us choose $h=\left(0,0,0, h^{3}\right)$. By an appropriate $S O(1,2)$ Lorentz transformation one can put $p=(\omega, 0, \omega, 0)$. The transversality condition imposes that $n_{0 \mu}=n_{2 \mu}$. Moreover (61) leads to $n_{3 \mu}=0$. The residual gauge invariance with $\epsilon^{3}=0$ allows one to put $n_{00}=n_{01}=0$. The polarisation matrix vanishes altogether. One can also understand this result by noticing that the polarisation condition and the restricted gauge invariance imply that massless gravitons effectively behave like $(1+2)$ massless gravitons. In three dimensions, a traceless transverse symmetric matrix has two independent degrees of freedom which are eaten by the two residual gauge invariances.

In conclusion we have seen that rotated and parallel branes are classical vacua of five dimensional supergravity with a non vanishing even part of the prepotential. Poincaré invariance is broken down to three dimensional Poincaré invariance implying that no massless gravitons propagate in the bulk.

\section{Supersymmetry Breaking}

In this section we will examine the spontaneous breaking of supersymmetry due to the detuning of the brane tension and the corresponding rotation of the boundary branes with respect to the $A d S_{5}$ bulk. We will first consider the Killing spinors and then the gravitini in the bulk.

\subsection{Killing Spinors}

The brane configuration preserves supersymmetry provided one can find Killing spinors compatible with the boundary conditions due to the $Z_{2}$ orbifold sym-

metry [17. Imposing that the supersymmetric variation $\delta \psi_{a}^{A}=0, a=1 \ldots 5$, $A=1,2$ implies that

$$
D_{a} \epsilon^{A}-\frac{i \sqrt{2}}{3} \gamma_{a} \mathcal{P}_{B}^{A} \epsilon^{B}=0
$$

where we are considering the bulk metric in conformal coordinates. This reduces to

$$
\partial_{a} \epsilon^{A}+\frac{k a}{2} \gamma_{a}^{5} \epsilon^{A}+\frac{k a}{2} \gamma_{a}\left(T \mathcal{Q}_{B}^{A}+\sqrt{1-T^{2}} \Sigma_{B}^{A}\right) \epsilon^{B}=0
$$


written with flat space Dirac matrices now. Let us introduce the projectors

$$
\Omega_{ \pm B}^{A}=\frac{1}{2}\left[\delta_{B}^{A} \pm \gamma_{5}\left(T \mathcal{Q}_{B}^{A}+\sqrt{1-T^{2}} \Sigma_{B}^{A}\right)\right]
$$

and the eigenspinors

$$
\Omega_{ \pm B}^{A} \epsilon_{ \pm}^{B}= \pm \epsilon_{ \pm}^{A}
$$

Now projecting (64) for $a=5$ on both chiralities and considering the following ansatz

$$
\epsilon_{ \pm}^{A}\left(x^{\mu}, y\right)=f_{ \pm}(k y) \epsilon_{ \pm}^{A}\left(x^{\mu}\right)
$$

yields

$$
\frac{d f_{ \pm}}{d y} \pm \frac{1}{2 y} f_{ \pm}=0
$$

and therefore the profile of the Killing spinors

$$
f_{ \pm}(y)=a^{ \pm 1 / 2}(y)
$$

Notice that the two types of Killing spinors are either peaked on the first or on the second brane. Let us now use the remaining Killing spinor equations for $a=\mu$

$$
\partial_{\mu} \epsilon^{A}-k a(y) \gamma_{\mu} \gamma^{5} \Omega_{-B}^{A} \epsilon^{B}=0 . .
$$

Projecting again on both chiralities and using the explicit dependence of $\epsilon_{ \pm}$on $y$, we find that

$$
\begin{aligned}
& \epsilon_{-}\left(x^{\mu}\right)=\epsilon_{-}^{0} \\
& \epsilon_{+}\left(x^{\mu}\right)=\epsilon_{+}^{0}+k x^{\mu} \gamma_{\mu} \gamma^{5} \epsilon_{-}^{0}
\end{aligned}
$$

where $\epsilon_{ \pm}^{0}$ are space-time independent and we have omitted the symplectic indices. The corresponding four dimensional Majorana spinors are defined as follows :

$$
\epsilon_{+}^{1}=f_{+}(y) \epsilon_{R}^{+}\left(x^{\mu}\right), \epsilon_{+}^{2}=f_{+}(y) \epsilon_{L}^{+}\left(x^{\mu}\right)
$$

and

$$
\epsilon_{-}^{1}=f_{-}(y) \epsilon_{L}^{-}\left(x^{\mu}\right), \epsilon_{-}^{2}=-f_{-}(y) \epsilon_{R}^{-}\left(x^{\mu}\right) .
$$

Let us now examine the compatibility with the boundary condition (22), evaluated on both branes and depending on the matrices $\Gamma=T\left(\gamma^{5}-h_{\mu} \gamma^{\mu}\right)$. After a $S U(2)_{R}$ rotation sending $\mathcal{Q}$ in the real $\left(\sigma_{1}, \sigma_{3}\right)$ plane and $T \mathcal{Q}+\sqrt{1-T^{2}} \Sigma \rightarrow \sigma_{3}$, we find that $\mathcal{Q}_{1}{ }_{1}=T$ and $\mathcal{Q}^{1}{ }_{2}=\varepsilon \sqrt{1-T^{2}}, \varepsilon= \pm$. Defining the normalized vector $u_{\mu}=\frac{T}{\sqrt{1-T^{2}}} h_{\mu}$ if $T \neq 1$, we find that the boundary condition reduces to

$$
f_{-}(y) \epsilon^{-}\left(x^{\mu}\right)=-\varepsilon \frac{\sqrt{1-T^{2}}}{T} \frac{1+\varepsilon u \cdot \gamma}{2} f_{+}(y) \epsilon^{+}\left(x^{\mu}\right)
$$

evaluated on both branes. This implies that

$$
\epsilon^{-}\left(x^{\mu}\right)=0,(1+\varepsilon u \cdot \gamma) \epsilon^{+}\left(x^{\mu}\right)=0,
$$


i.e. only two components of the spinor $\epsilon^{+}$survive the projection. Thus only $1 / 4$ of the original eight supersymmetries are conserved by the vacuum for $T<1$ parallel branes. The existence of configurations with less than four supersymmetries in $4 \mathrm{~d}$ has already been considered in 30. The explicit appearance of the non-zero $u_{\mu}$ signals a breaking of $4 \mathrm{~d}$ Lorentz invariance too.

In the case $T=1$ with non-zero light-like tilting vector $h_{\mu}$, we have on each brane instead of (74) :

$$
f_{-}(y) \epsilon_{-}\left(x^{\mu}\right)=-\frac{1}{2}(h \cdot \gamma) f_{+}(y) \epsilon_{+}\left(x^{\mu}\right) .
$$

This results in the (4d Lorentz violating) constraints

$$
\epsilon^{-}\left(x^{\mu}\right)=0,(h \cdot \gamma) \epsilon^{+}\left(x^{\mu}\right)=0 .
$$

This also preserves $1 / 4$ of the supersymmetries.

\subsection{Gravitini in the bulk}

Having realized that a generic tilted configuration breaks supersymmetry one can expect that the tower of Kaluza-Klein modes for the gravitini is going to be affected too. Let us consider the gravitino equation in the bulk first

$$
\gamma^{a b c} D_{b} \psi_{c}^{A}+\sqrt{2} i \mathcal{P}_{B}^{A} \psi_{c B}=0
$$

where we consider the supersymmetric gauge $\psi_{5}=0$. This can be reexpressed as

$$
\gamma^{\mu \nu \rho} \partial_{\nu} \psi_{\rho}^{A}-a \gamma^{5} \gamma^{\mu \nu} \partial_{5} \psi_{\rho}^{A}+\frac{5 k}{2} a \gamma^{5} \gamma^{\nu \rho} \psi_{\rho}^{A}-3 k a \gamma^{5} \gamma^{\mu \nu}\left(\Omega_{+B}^{A} \psi_{\rho}^{B}\right)=0
$$

for $a=\mu$ and the gamma matrices are associated to the Minkowski metric here. Let us perform as before a $S U(2)_{R}$ rotation to send $\Omega_{ \pm}$to

$$
\Omega_{ \pm B}^{A} \rightarrow \frac{1}{2}\left(\delta_{B}^{A} \pm \gamma^{5}\left(\sigma_{3}\right)_{B}^{A}\right)
$$

and decompose the symplectic spinors according to

$$
\left(\Omega_{+} \psi_{\rho}\right)^{1}=\phi_{+}\left(x_{5}\right) \psi_{\rho R}^{+}\left(x^{\mu}\right),\left(\Omega_{+} \psi_{\rho}\right)^{2}=\phi_{+}\left(x_{5}\right) \psi_{\rho L}^{+}\left(x^{\mu}\right)
$$

and

$$
\left(\Omega_{-} \psi_{\rho}\right)^{1}=\phi_{-}\left(x_{5}\right) \psi_{\rho L}^{-}\left(x^{\mu}\right),\left(\Omega_{-} \psi_{\rho}\right)^{2}=-\phi_{-}\left(x_{5}\right) \psi_{\rho R}^{-}\left(x^{\mu}\right)
$$

where the two spinors $\psi^{ \pm}$are four dimensional Majorana spinors. Due to the symplectic features of the five dimensional spinors, it is sufficient to consider the $A=1$ equation only. Moreover we are looking for massive gravitini from the four dimensional point of view

$$
\gamma^{\mu \nu \rho} \partial_{\nu} \psi_{\rho}^{ \pm}=m \gamma^{\mu \rho} \psi_{\rho}^{\mp} .
$$


Notice the change of chirality. The gravitino profiles satisfy

$$
\begin{aligned}
\frac{m}{a} \phi_{-} & =\partial_{5} \phi_{+}+\frac{k}{2} \phi_{+} \\
\frac{m}{a} \phi_{+} & =-\partial_{5} \phi_{-}+\frac{5 k}{2} \phi_{-} .
\end{aligned}
$$

These are the same differential equations as for the supersymmetric RandallSundrum model [19. Using the $a=5$ equation we obtain the irreducibility constraint

$$
\gamma^{\mu} \psi_{\mu}^{-}=0
$$

for the massless gravitino $\psi_{\mu}^{-}$.

Let us now consider the compatibility with the boundary condition

$$
\left.\left(\delta_{B}^{A}-\Gamma \mathcal{Q}_{B}^{A}\right) \psi_{\mu}^{B}\right|_{\text {branes }}=0 .
$$

The discussion is exactly the same as for the Killing spinors. For proportional tilting vectors, $1 / 4$ of them would survive the projection :

$$
\begin{array}{cl}
\psi_{\mu}^{-}=0 & \text { and } \\
(1+\varepsilon u \cdot \gamma) \psi_{\mu}^{+}=0 & \text { for } T<1 \\
(h \cdot \gamma) \psi_{\mu}^{+}=0 & \text { for } T=1, h_{\mu} \neq 0 .
\end{array}
$$

Combined with the Rarita-Schwinger equation connecting the two spinors $\psi_{\mu}^{ \pm}$ this leads to

$$
\psi_{\mu}^{ \pm}=0
$$

in the massive case. Only massless gravitini survive in agreement with the $1 / 4$ of remaining supersymmetries.

In conclusion we have found that parallel branes are vacua of spontaneously broken $5 \mathrm{~d}$ supergravity preserving only $1 / 4$ of supersymmetry and $3 \mathrm{~d}$ Poincaré invariance.

\section{The Low Energy Action}

\subsection{Radion instability}

So far we have only considered parallel and rotated brane vacua of $5 \mathrm{~d}$ supergravity and shown that supersymmetry is broken in that case. We have also discarded other vacua which lead to brane intersections where the supergravity description breaks down. In this section we will use the low energy effective action describing the dynamics of the moduli fields, i.e. the graviton and the radion in the bosonic sector. This allows one to analyse a wide range of possible vacua including time dependent configurations. Nevertheless, the rotated branes may be out of reach of the low energy approximation due to their nonperturbative nature compared to the Randall- Sundrum straight branes. We 
will analyse the static solutions of the low energy equations of motion and find configurations which are locally similar to the rotated branes.

As an example, one can embed the two boundary branes in an $A d S_{5}$-Schwarzschild background with black-hole mass $\mu$ and $3 \mathrm{~d}$ sectional curvature $q$ to find that the branes move according to

$$
H^{2}=\left(T^{2}-1\right) k^{2}-\frac{q}{r^{2}}+\frac{\mu}{r^{4}}
$$

where $H=\dot{r} / r$. These backgrounds break supersymmetry too 31. More generally the brane geometry is described within the projective approach 32 where the brane Einstein equations depend on the matter content of each brane and the projected Weyl tensor. The latter is bulk dependent and reduces to the black hole mass in the time-dependent setting. In general, one does not know how to describe the general solutions of the boundary brane Einstein equations. On the contrary when the matter content of the branes is a small perturbation compared to the brane tension of the Randall-Sundrum $T=1$ case, one can use the moduli space approximation to understand the dynamics fully.

This description is known to coincide with the projective approach as long as the deviation of the brane tension from the BPS $(T=1)$ case is small, i.e. when $|T-1|<<1[33$. The fact that $T \neq 1$ leads to a non vanishing potential at low energy for the radion field. We will analyse the solutions of the equations of motion and show that either the configuration ends up in a cosmological big crunch singularity or the radion vanishes in a locus where the supergravity description breaks down.

In the moduli space approach, one considers the bulk metric

$$
d s^{2}=a^{2}\left(x_{5}\right) g_{\mu \nu}\left(x^{\mu}\right) d x^{\mu} d x^{\nu}+d x_{5}^{2}
$$

and the branes are located at 0 and $R\left(x^{\mu}\right)$. Integrating over the fifth dimension, the dynamics of the branes can be described by an effective action involving the radion and determined by the underlying supergravity Kähler potential [20, 21]

$$
K=-3 \ln \left(1-e^{-k(S+\bar{S})}\right)
$$

where $S$ is the radion superfield and $S+\bar{S}=2 R$ for its bosonic part. This specifies the action entirely up to the supersymmetry breaking potential induced by the detuning of the brane tensions. The potential term is

$$
\frac{6 k(1-T)}{\kappa_{5}^{2}} \int d^{4} x \sqrt{-g}\left(1-e^{-4 k R}\right)
$$

in the brane frame where the Einstein-Hilbert term reads

$$
\frac{1}{4 k \kappa_{5}^{2}} \int d^{4} x \sqrt{-g}\left(1-e^{-2 k R}\right) \mathcal{R}
$$

and $\mathcal{R}$ is the $4 \mathrm{~d}$ curvature. In the Einstein frame the radion action reads

$$
-\frac{k}{4 \kappa_{5}^{2}} \int d^{4} x \sqrt{-g}\left(\frac{6 e^{2 k R}}{k^{4}\left(e^{2 k R}-1\right)^{2}}(\partial R)^{2}+24(T-1) \frac{\left(1-e^{-4 k R}\right)}{\left(1-e^{-2 k R}\right)^{2}}\right) .
$$


It is convenient to normalise the radion kinetic terms by defining

$$
\phi=\frac{1}{2} \ln \left(\frac{e^{k R}-1}{e^{k R}+1}\right)
$$

in such a way that the action becomes

$$
-\frac{k}{4 \kappa_{5}^{2}} \int \sqrt{-g}\left(\frac{6}{k^{2}}(\partial \phi)^{2}+12(T-1) \cosh (2 \phi)\right) .
$$

Notice that $\phi$ varies between $-\infty$ and zero. The potential is unbounded from below and always negative.

Let us take the limit $\phi=0$ where the branes are infinitely far apart, i.e. the one brane system. The potential reduces to a cosmological constant

$$
\Lambda_{\phi=0}=\frac{3(T-1) k}{\kappa_{5}^{2}}
$$

This cosmological constant for a one-brane system coincides with the boundary cosmological constant as obtained from the $A d S_{4}$ boundary metric

$$
\Lambda_{A d S_{4}}=\frac{3\left(T^{2}-1\right) k^{2}}{\kappa_{4}^{2}}
$$

for $|T-1|<<1$ and we have used $\kappa_{4}^{2}=2 k \kappa_{5}^{2}$. The two-brane system has a non trivial potential whose limiting value is equal to the one-brane cosmological constant.

Let us now show that cosmological solutions of this low energy action always reach a big crunch singularity. We focus on flat spatial sections. Looking for FRW solutions in the Einstein frame with scale factor $a$ leads to

$$
\frac{\ddot{a}}{a}=-4\left(\frac{(\dot{\phi})^{2}}{k^{2}}+(1-T) \cosh (2 \phi)\right)
$$

in $4 \mathrm{~d}$ Planck units and ${ }^{*}=d / d t$. Notice that $\ddot{a}<0$ implying that $\dot{a}$ decreases. Starting from $\dot{a}>0$, the scale factor first increases before reaching a turning point where $\dot{a}=0$, then entering a contracting phase with $\dot{a}<0$. Eventually the scale factor has to vanish as no minimum for $a$ can exist. Hence cosmological solutions with a flat spatial section end up in a big crunch singularity.

Consider static configurations now. We choose the ansatz for the metric

$$
g_{\mu \nu} d x^{\mu} d x^{\nu}=d x_{3}^{2}+a^{2}\left(x_{3}\right)\left(-d t^{2}+d \Omega_{\tilde{k}}^{2}\right)
$$

where $d \Omega_{\tilde{k}}^{2}$ is the line element of the two dimensional symmetric space with curvature $\tilde{k}=0, \pm 1$. We also restrict ourselves to $\phi\left(x_{3}\right)$. Define

$$
\tilde{V}=12 k^{2}(1-T) \cosh \frac{\tilde{\phi}}{\sqrt{3}}
$$


where $\tilde{\phi}=2 \sqrt{3} \phi$ is the canonically normalised field. Notice that $\tilde{V}$ is the opposite of the potential appearing in the action. The Einstein equations lead to the Friedmann equation

$$
H^{2}=\frac{1}{6}\left(\frac{\tilde{\phi}^{\prime 2}}{2}+\tilde{V}\right)+\frac{\tilde{k}}{3 a^{2}}
$$

We have introduced $H=\frac{a^{\prime}}{a}$ where $^{\prime}=d / d x_{3}$. Notice the factor of 3 in the curvature term as we only consider two dimensional static symmetric spaces. The Klein-Gordon reads

$$
\tilde{\phi}^{\prime \prime}+3 H \tilde{\phi}^{\prime}=-\frac{\partial \tilde{V}}{\partial \tilde{\phi}}
$$

We have thus rewritten the equations of motion of static configurations in a form similar to cosmological equations where time is replaced by $x_{3}$.

We are looking for solutions where the branes at infinity $x_{3}= \pm \infty$ in the coordinate system used to define the metric (100) are far away $\phi=0$. This corresponds to the field starting at the minimum of the potential, the field being negative decreases and reaches a minimum before falling down to the origin again. We will consider the case where the minimum is close to the origin and $\phi^{\prime}$ remains small. In that case the Friedmann equation reduces to

$$
H^{2}=-\frac{\Lambda_{A d S_{4}}}{3}+\frac{\tilde{k}}{3 a^{2}}
$$

Choosing $\tilde{k}=-1$, we find a bounce

$$
a\left(x_{3}\right)=\frac{1}{\sqrt{-\Lambda_{A d S_{4}}}} \cosh \left(\sqrt{\frac{-\Lambda_{A d S_{4}}}{3}} x_{3}\right)
$$

We can now discuss the solutions of the Klein-Gordon equation in two regimes. When $\left|\sqrt{\frac{-\Lambda_{A d S_{4}}}{3}} x_{3}\right|>>1$, one can approximate $H^{2}=\frac{-\Lambda_{A d S_{4}}}{3}$ and the KleinGordon equation becomes

$$
\tilde{\phi}^{\prime \prime}+3 H \tilde{\phi}^{\prime}+2 H^{2} \tilde{\phi}=0
$$

Hence the field $\tilde{\phi}$ behaves like

$$
\tilde{\phi}=A_{-} e^{\sqrt{\frac{-\Lambda_{A d S_{4}}}{3}} x_{3}}+B_{-} e^{2 \sqrt{\frac{-\Lambda_{A d S_{4}}}{3}} x_{3}}
$$

for $x_{3}<0$ and

$$
\tilde{\phi}=A_{+} e^{-\sqrt{\frac{-\Lambda}{A d S_{4}}} x_{3}}+B_{+} e^{-2 \sqrt{\frac{-\Lambda A d S_{4}}{3}} x_{3}}
$$

for $x_{3}>0$ and some constants $A_{ \pm}<0$ and $B_{ \pm}<0$. When $\left|\sqrt{\frac{-\Lambda_{A d S_{4}}}{3}} x_{3}\right|<<1$ one can neglect $H$ in the Klein-Gordon equation leading to

$$
\tilde{\phi}=C \cos \left(\sqrt{\frac{-2 \Lambda_{A d S_{4}}}{3}} x_{3}\right)
$$


for some constant $C$. It is interesting to connect $\tilde{\phi}$ and the radion. For large $\left|x_{3}\right|$ the radion is linear

$$
R\left(x_{3}\right)=h\left|x_{3}\right|
$$

while it is goes to a non-vanishing constant for small $x_{3}$. Hence we find that the second brane is wedge-like. Locally this solution resembles the rotated branes obtained previously although in a different coordinate system.

For phenomenological models, this type of behaviour for the radion is particularly undesirable as it leads to large Lorentz violating effects due to the spatial dependence of the radion field. It also leads to a negative potential energy as compared to the observation of a small positive cosmological constant. Of course this prompts towards modifying the previous setting by including other sources of supersymmetry breaking which may compensate the negative radion potential that we have derived.

\subsection{Charged Branes}

So far we have considered neutral branes. Here we will consider charged branes. One can also introduce a bulk four-form $C$ coupling to the branes

$$
S=-\frac{1}{2} \int F \wedge * F-\frac{2 \kappa_{5}}{\sqrt{3}} Q \int_{+} C+\frac{2 \kappa_{5}}{\sqrt{3}} Q \int_{-} C
$$

where $Q$ is the charge of the positive tension brane denoted by $+(-$ is the negative tension brane). We have defined the field strength

$$
F=d C
$$

and the dual field $* F$ is a scalar, hence the wedge product is not necessary in the kinetic terms. The equations of motion are given by

$$
\partial_{5}(* F)=\frac{2 \kappa_{5}}{\sqrt{3}} Q\left(\delta_{0}-\delta_{R}\right)
$$

implying that

$$
* F=\frac{\kappa_{5}}{\sqrt{3}} Q \epsilon\left(x_{5}\right) .
$$

By duality we find that

$$
F=\frac{\kappa_{5}}{\sqrt{3}} Q \epsilon\left(x_{5}\right) \sqrt{-g_{5}} d x^{1} \wedge d x^{2} \wedge d x^{3} \wedge d x^{4} \wedge d x^{5} .
$$

Notice that $F$ is odd on the orbifold implying that its integral, i.e. the boundary terms, in the action vanishes identically. Substituting in the action and integrating over the extra-dimension we find

$$
-\frac{\kappa_{5}^{2} Q^{2}}{12 k} \int d^{4} x \sqrt{-g}\left(1-e^{-4 k R}\right) .
$$


In the Einstein frame, this leads to a potential for the radion

$$
V_{F}=\frac{\kappa_{5}^{2} Q^{2}}{12 k} \cosh 2 \phi .
$$

The total potential energy including both the effects of detuning the brane tensions and the coupling to the bulk four-form reads

$$
V=\left(\frac{\kappa_{5}^{2} Q^{2}}{12 k}-\frac{3(1-T) k}{\kappa_{5}^{2}}\right) \cosh 2 \phi
$$

We find that there is a BPS bound

$$
\left|Q_{B P S}\right|=\frac{6(1-T) k}{\kappa_{5}^{2}}
$$

where the charge of the brane is equal to the deficit of brane tension. This case might be a necessary condition to allow the supersymmetrization of the 4form action, once proper superpartners have been introduced, although we have not tried to ascertain this result. Below the BPS bound, the radion potential is unbounded from below. When the charge saturates the BPS bound, we see immediately that the radion potential vanishes altogether, i.e. the radion remains a flat direction. This signals the absence of force between the branes. Above the BPS bound the radion acquires a positive potential. The radion rolls down towards infinity where the branes are infinitely far apart. Doing so, the asymptotic dynamics are dominated by a cosmological constant. In conclusion, the brane system is not stabilised once supersymmetry is broken. In the most favourable case above the BPS bound, the two branes repulse each other.

\section{Conclusion}

We have presented results on the spontaneous breaking of supersymmetry in five dimensional supergravity with two boundaries. We have studied classical configurations corresponding to rotated branes with either singular intersecting branes or parallel branes preserving $1 / 4$ of supersymmetry. We have then analysed the low energy effective action involving the coupling of the radion field to $4 \mathrm{~d}$ gravity. The radion acquires a negative and unbounded from below potential. The radion potential can be rendered bounded from below by coupling the boundary branes with a four-form. Above a BPS bound, the potential admits a minimum corresponding to infinitely separated branes. When saturating the BPS bound linking the charge to the deficit of tension, the radion has a flat potential. Above the BPS bound the branes roll away to infinity where the remaining cosmological constant is positive. The phenomenology of such a system is currently under study.

\section{Acknowledgments}

We would like to thank C. van de Bruck, C. Charmousis, C. Grojean, Z. Lalak, J. Mourad and F. Quevedo for useful discussions and comments. 


\section{Appendix A: Rotated branes and $A d S_{4}$ branes}

Consider a single detuned brane $T<1$. One can find a solution of the equations of motion where slices of constant $z$ admit an $A d S_{4}$ metric

$$
d s^{2}=a^{2}(z) g_{\mu \nu} d \tilde{x}^{\mu} d \tilde{x}^{\nu}+d z^{2}
$$

where $g_{\mu \nu}$ is the $A d S_{4}$ metric with cosmological constant

$$
\Lambda_{A d S_{4}}=3\left(T^{2}-1\right) k^{2}<0
$$

and

$$
g_{\mu \nu} d \tilde{x}^{\mu} d \tilde{x}^{\nu}=e^{-2 \sqrt{-\frac{1}{3} \Lambda_{A d S_{4}}} \tilde{x}_{3}}\left(-d \tilde{t}^{2}+d \tilde{x}_{1}^{2}+d \tilde{x}_{2}^{2}\right)+d \tilde{x}_{3}^{2} .
$$

The scale factor reads

$$
a(z)=\frac{\sqrt{-\frac{1}{3} \Lambda_{A d S_{4}}}}{k} \cosh (k z-C) .
$$

The metric is isometric to the warped metric of $A d S_{5}$

$$
d s^{2}=e^{-2 k x_{5}} \eta_{\mu \nu} d x^{\mu} d x^{\nu}+d x_{5}^{2}
$$

using

$$
\begin{aligned}
& x_{3}=\sqrt{-\frac{3}{\Lambda_{A d S_{4}}}} \tanh (C-k z) e^{\sqrt{-\frac{1}{3} \Lambda_{A d S_{4}}} \tilde{x}_{3}} \\
& x_{5}=\sqrt{-\frac{\Lambda_{A d S_{4}}}{3}} \frac{\tilde{x}_{3}}{k}-\frac{\ln \left(\frac{-\Lambda_{A d S_{4}}}{3 k} \cosh (C-k z)\right)}{k}
\end{aligned}
$$

and $\tilde{t}=t, \tilde{x}_{1}=x_{1}, \tilde{x}_{2}=x_{2}$. The boundary condition at $z=0$ for the single brane reads

$$
\tanh (C)=T \text {. }
$$

Under the previous mapping, the brane at $z=0$ is mapped to a rotated brane

$$
y=h x_{3}
$$

in conformal coordinates $k y=e^{k x_{5}}$. Now let us try to include a second brane with negative tension at $z=\rho$, the boundary condition reads

$$
\tanh (k \rho-C)=-T
$$

whose only solution is $\rho=0$. Hence the metric with $A d S_{4}$ slicing at constant $z$ is not a solution of the two brane system. 


\section{Appendix B: Massless Gravitons}

Let us give a thorough analysis of the Israel matching conditions applied to the massless gravitons. We choose to work in the transverse traceless gauge where

$$
n_{\mu \nu}\left(y, x^{\mu}\right)=\phi(y) e^{i p . x} n_{\mu \nu} .
$$

The condition (57) reduces to

$$
h_{\lambda}\left(p_{\mu} n_{\nu}^{\lambda}+p_{\nu} n_{\mu}^{\lambda}-p^{\lambda} n_{\mu \nu}\right)=a k \frac{h_{\mu} n^{\mu \nu} h_{\nu}}{1+h^{2}}\left(\eta_{\mu \nu}+h_{\mu} h_{\nu}\right) .
$$

Contracting with $p^{\mu}$ and using $p^{2}=0$ one finds that

$$
h_{\mu} n^{\mu \nu} h_{\nu}=0
$$

together with

$$
h_{\lambda}\left(p_{\mu} n_{\nu}^{\lambda}+p_{\nu} n_{\mu}^{\lambda}-p^{\lambda} n_{\mu \nu}\right)=0 .
$$

This equation can be treated separating two different cases.

\section{$\mathbf{9 . 1} p . h \neq 0$}

Let us choose two space-like orthogonal unit vectors $e_{i}^{\mu}, i=1,2$, which are orthogonal to $p$ and $h$. This completes a basis of $4 \mathrm{~d}$ vectors. Projecting the boundary equation (132) leads to

$$
e_{i} . n . e_{j}=0 .
$$

Let us now choose a particular basis of traceless and symmetric tensors comprising $p^{\mu} p^{\nu},\left(h^{\mu} h^{\nu}-\frac{h^{2}}{4} \eta^{\mu \nu}\right),\left(e_{i}^{\mu} e_{i}^{\nu}-\frac{1}{4} \eta^{\mu \nu}\right), \frac{1}{2}\left(e_{1}^{\mu} e_{2}^{\nu}+e_{1}^{\nu} e_{2}^{\mu}\right), \frac{1}{2}\left(e_{i}^{\mu} h^{\nu}+e_{i}^{\nu} h^{\mu}\right)$ and $\frac{1}{2}\left(e_{i}^{\mu} p^{\nu}+e_{i}^{\nu} p^{\mu}\right)$. Using the transverse condition and (131133), one finds that

$$
n^{\mu \nu}=\sum_{i=1}^{2} \frac{\alpha_{i}}{2}\left(e_{i}^{\mu} p^{\nu}+e_{i}^{\nu} p^{\mu}\right) .
$$

Using the residual gauge invariance with vector $\epsilon^{\mu} e^{i p . x}$ such that

$$
\epsilon^{\mu}=\sum_{i=1}^{2} \beta_{i} e_{i}^{\mu}
$$

the gauge transformation

$$
n_{\mu \nu} \rightarrow n_{\mu \nu}+p_{\mu} \epsilon_{\nu}+p_{\nu} \epsilon_{\mu}
$$

one can fix $\beta_{i}=-\frac{1}{2} \alpha_{i}$. Hence no massless graviton appears in the spectrum. 


\section{$9.2 \quad p . h=0$}

Notice first that one can choose a basis of vectors such that $e_{i}^{\mu}$ are now such that $h . e_{i}=0, e_{1} \cdot e_{2}=0$ and $p . e_{2}=0$. Notice that $p . e_{1} \neq 0$ here. The new basis of traceless symmetric tensors is now $p^{\mu} p^{\nu},\left(h^{\mu} h^{\nu}-\frac{h^{2}}{4} \eta^{\mu \nu}\right),\left(e_{i}^{\mu} e_{i}^{\nu}-\frac{1}{4} \eta^{\mu \nu}\right)$, $\frac{1}{2}\left(e_{1}^{\mu} e_{2}^{\nu}+e_{1}^{\nu} e_{2}^{\mu}\right), \frac{1}{2}\left(e_{i}^{\mu} h^{\nu}+e_{i}^{\nu} h^{\mu}\right), \frac{1}{2}\left(e_{2}^{\mu} p^{\nu}+e_{2}^{\nu} p^{\mu}\right)$ and $\frac{1}{2}\left(p^{\mu} h^{\nu}+p^{\nu} h^{\mu}\right)$. The boundary equation reads now

$$
h_{\lambda}\left(p_{\mu} n_{\nu}^{\lambda}+p_{\nu} n_{\mu}^{\lambda}\right)=0
$$

leading to

$$
e_{i}^{\mu} n_{\mu \nu} h^{\nu}=0
$$

The solutions to these constraints read now

$$
n_{\mu \nu}=\alpha p_{\mu} p_{\nu}+\frac{\alpha_{2}}{2}\left(e_{2}^{\mu} p^{\nu}+e_{2}^{\nu} p^{\mu}\right)
$$

The residual gauge invariance parametrized by $\epsilon^{\mu} e^{i p . x}$ involves two components

$$
\epsilon^{\mu}=\beta_{2} e_{2}^{\mu}+\beta p^{\mu} .
$$

Using $\beta=-\frac{1}{2} \alpha$ and $\beta_{2}=-\frac{1}{2} \alpha_{2}$, no graviton remains in the spectrum.

\section{References}

[1] J. Polchinski, String theory, Cambridge University Press (1998).

[2] F. Quevedo, Gaugino Condensation, Duality and Supersymmetry Breaking, Nucl.Phys.Proc.Suppl. 46 (1996) 187-197 arXiv:hep-th/9511131; F. Quevedo, Lectures on Superstring Phenomenology, In *Puebla 1995, Particles and fields and phenomenology of fundamental interactions* 202-242 arXiv:hep-th/9603074 ; M. Dine, Supersymmetry Phenomenology With a Broad Brush, in *Boulder 1996, Fields, strings and duality* 813-881 arXiv:hep-ph/9612389.

[3] M. Dine and Y. Shirman, Remarks on the Racetrack Scheme, Phys.Rev. D 63 (2001) 046005 arXiv:hep-th/9906246 ; R. Ciesielski and Z. Lalak, Racetrack models in theories from extra dimensions, JHEP 0212 (2002) 028 arXiv:hep-ph/0206134.

[4] A. G. Riess et al, Observational Evidence from Supernovae for an Accelerating Universe and a Cosmological Constant, Astron.J. 116 (1998) 1009-1038 arXiv:astro-ph/9805201.

[5] I. Antoniadis, C. Muñoz, M. Quirós, Dynamical supersymmetry breaking with a large internal dimension, Nucl.Phys. B 397 (1993) 515-538, arXiv:hep-ph/9211309 ; I. Antoniadis, A. Sagnotti, Mass scales, supersymmetry breaking and open strings, Class.Quant.Grav. 17 (2000) 939-950 arXiv:hep-th/9911205. 
[6] J. Polchinski, Dirichlet-Branes and Ramond-Ramond Charges, Phys.Rev.Lett. 75 (1995) 4724-4727 arXiv:hep-th/9510017.

[7] P. Horava, Gluino Condensation in Strongly Coupled Heterotic String Theory, Phys. Rev. D 54 (1996) 7561-7569 arXiv:hep-th/9608019 ; E.A. Mirabelli and M.E. Peskin, Transmission of supersymmetry breaking from a 4-dimensional boundary, Phys. Rev. D 58 (1998) 065002 arXiv:hep-th/9712214 ; L. Randall and R. Sundrum, Out of this world supersymmetry breaking, Nucl. Phys. B 557 (1999) 79-118 arXiv:hep-th/9810155.

[8] A. Sen, Non-BPS States and Branes in String Theory arXiv:hep-th/9904207.

[9] M. Fabinger and P. Horava, Casimir effect between world-branes in heterotic M-theory, Nucl. Phys. B 580 (2000) 243 arXiv:hep-th/0002073.

[10] P. Brax, A. Falkowski and Z. Lalak, Non-BPS branes of supersymmetric brane worlds, Phys. Lett. B 521 (2001) 105 arXiv:hep-th/0107257.

[11] P. Brax, Non-BPS instability in heterotic M-theory, Phys. Lett. B 506 (2001) 362-368, Phys. Lett. B 508 (2001) 375-380 arXiv:hep-th/0102154.

[12] W. D. Linch III, M. A. Luty, J. Phillips, Phys. Rev. D68 (2003), 025008, arXiv:hep-th/0209060; I. L. Buchbinder, S. J. Gates, H. S. Goh, W. D. Linch III, M. A. Luty, S. P. Ng, J. Phillips arXiv:hep-th/0305169.

[13] L. Randall and R. Sundrum, A large mass hierarchy from a small extra dimension, Phys. Rev. Lett. 83 (1999) 3370 arXiv:hep-ph/9905221.

[14] L. Randall and R. Sundrum, An alternative to compactification, Phys. Rev. Lett. 83 (1999) 4690 arXiv:hep-th/9906064.

[15] R. Altendorfer, J. Bagger and D. Nemeschansky, Supersymmetric RandallSundrum scenario, Phys. Rev. D 63 (2001) 125025 arXiv:hep-th/0003117.

[16] A. Falkowski, Z. Lalak and S. Pokorski, Supersymmetrizing branes with bulk in five-dimensional supergravity, Phys. Lett. B 491 (2000) 172 arXiv:hep-th/0004093.

[17] E. Bergshoeff, R. Kallosh and A. Van Proeyen, Supersymmetry in singular spaces, JHEP 0010 (2000) 033 arXiv:hep-th/0007044.

[18] M. Zucker, Supersymmetric brane world scenarios from off-shell supergravity, Phys. Rev. D 64 (2001) 024024 arXiv:hep-th/0009083.

[19] A. Falkowski, Z. Lalak and S. Pokorski, Five-dimensional gauged supergravities with universal hypermultiplet and warped brane worlds, Phys. Lett. B 509 (2001) 337 arXiv:hep-th/0009167. 
[20] A. Falkowski, Z. Lalak and S. Pokorski, Four dimensional supergravities from five dimensional brane worlds, Nucl. Phys. B 613 (2001) 189-217 arXiv:hep-th/0102145.

[21] J. Bagger, D. Nemeschansky and R.-J. Zhang, Supersymmetric radion in the Randall-Sundrum scenario, JHEP 0108 (2001) 057 arXiv:hep-th/0012163.

[22] J. Bagger and D. Belyaev, Supersymmetric branes with (almost) arbitrary tensions, Phys. Rev. D 67 (2003) 025004 arXiv:hep-th/0206024.

[23] P. Brax and Z. Lalak, Brane world supersymmetry, detuning, flipping and orbifolding, Acta Phys. Polon. B 33 (2002) 2399 arXiv:hep-th/0207102.

[24] Z. Lalak and R. Matyszkiewicz, On Scherk-Schwarz mechanism in gauged five-dimensional supergravity and on its relation to bigravity, Nucl. Phys. B 649 (2003) 389 arXiv:hep-th/0210053.

[25] Z. Lalak and R. Matyszkiewicz, Twisted supergravity and untwisted superbigravity, Phys.Lett. B 562 (2003) 347-357 arXiv:hep-th/0303227.

[26] J. Bagger and D. Belyaev, Twisting Warped Supergravity, JHEP 0306 (2003) 013 arXiv:hep-th/0306063.

[27] A. Lukas, B. A. Ovrut, K.S. Stelle and D. Waldram, Heterotic M-theory in Five Dimensions, Nucl. Phys. B 552 (1999) 246 arXiv:hep-th/9806051.

[28] C. Charmousis, R. Gregory and V. A. Rubakov, Wave function of the radion in a brane world, Phys. Rev. D 62 (2000) 067505 arXiv:hep-th/9912160 ; P. Brax, C. van de Bruck, A.C. Davis and C.S. Rhodes, Wave function of the radion with a bulk scalar field, Phys. Lett. B 531 (2002) 135-142 arXiv:hep-th/0201191.

[29] O. DeWolfe, D. Freedman, S. Gubser and A. Karch, Modeling the fifth dimension with scalars and gravity, Phys. Rev. D 62 (2000) 046008 arXiv:hep-th/9909134 ; A. Karch and L. Randall, Locally Localized Gravity, JHEP 0105 (2001) 008 hep-th/0011156.

[30] J. P. Gauntlett, G. W. Gibbons, C. M. Hull, P. K. Townsend, BPS States of $D=4 N=1$ supersymmetry, Commm. Math. Phys. 216 (2001), 431 [arXiv: hep-th/0001024.

[31] P. Brax, A. Falkowski and Z. Lalak, Cosmological constant and kinetic supersymmetry breakdown on a moving brane, Nucl.Phys.B 667 (2003) 149169 arXiv:hep-th/0303167.

[32] T. Shiromizu, K. Maeda and M. Sasaki, The Einstein Equations on the 3-Brane World, Phys.Rev. D 62 (2000) 024012 arXiv:gr-qc/9910076 ; M. Sasaki, T. Shiromizu and K. Maeda, Gravity, Stability and Energy Conservation on the Randall-Sundrum Brane-World, Phys.Rev. D 62 (2000) 
024008 arXiv:hep-th/9912233 ; S. Kanno and J. Soda, Brane World Effective Action at Low Energies and AdS/CFT Correspondence, Phys.Rev. D 66 (2002) 043526 arXiv:hep-th/0205188.

[33] S. Kanno and J. Soda, Radion and Holographic Brane Gravity, Phys.Rev. D 66 (2002) 083506 arXiv:hep-th/0207029 ; T. Shiromizu and K. Koyama, Low energy effective theory for two branes system, Phys.Rev. D 67 (2003) 084022 arXiv:hep-th/0210066 ; S. Kanno, M. Sasaki and J. Soda, Born-Again Braneworld, Prog.Theor.Phys. 109 (2003) 357-369 arXiv:hep-th/0210250 ; T. Shiromizu, K. Koyama and K. Takahashi, Effective theory for close limit of two branes, Phys.Rev. D 67 (2003) 104011 arXiv:hep-th/0212331 ; J. Soda and S. Kanno, Non-linear Gravity on Branes and Effective Action arXiv:gr-qc/0301125. 